\title{
EFICIÊNCIA DE FUNGICIDAS NO CONTROLE DA MANCHA PRETA E VERRUGOSE DO AMENDOIM POR MÉTODO DE MONITORAMENTO*
}

\author{
SÉRGIO A. MORAES ${ }^{1 * *}$, IGNÁCIO J. GODOY ${ }^{1 * *}$, JOSÉ R.M. PEZZOPANE ${ }^{1}$, JOSÉ C.V.N.A. PEREIRA ${ }^{1}$ \\ \& LUIZ C.P. SILVEIRA ${ }^{1}$
}

${ }^{1}$ Instituto Agronômico de Campinas, Caixa Postal 28, CEP 13.001-970, Campinas, SP, e-mail: sergioam@cec.iac.br

(Aceito para publicação em 02/01/2001)

Autor para correspondência: Sérgio Almeida de Moraes

MORAES, S.A., GODOY, I.J., PEZZOPANE, J.R.M., PEREIRA, J.C.V.N.A. \& SILVEIRA, L.C.P. Eficiência de fungicidas no controle da mancha preta e verrugose do amendoim por método de monitoramento. Fitopatologia Brasileira 26:134-140. 2001.

\section{RESUMO}

Visando avaliar a eficiência de quatro fungicidas no controle monitorado da mancha preta (Cercosporidium personatum) e verrugose (Sphaceloma arachidis) do amendoim (Arachis hypogaea), cv.'Tatu', foram conduzidos quatro ensaios em Ribeirão Preto e Pindorama, SP (1996 e 1997. Os tratamentos foram: testemunha, sem controle químico; controle convencional, com quatro pulverizações de clorotalonil, a cada 14 dias, iniciando entre 41 e 43 dias do plantio; e controle monitorado, com clorotalonil e os triazóis tebuconazole, difenoconazole e propiconazole, nas doses recomendadas pelos fabricantes para a cultura. No controle monitorado a primeira pulverização foi realizada quando a incidência da mancha preta foi de 5 a $15 \%$ e as demais, com o intervalo mínimo de 14 dias entre as aplicações, após três dias, seguidos ou não, com ocorrências de precipitações pluviais superiores a $2,5 \mathrm{~mm}$, durante períodos de sete dias. A intensidade da mancha preta (área sob a curva de progresso da doença) foi obtida por avaliações semanais com escalas diagramáticas de índice de área foliar infetada, durante o ciclo da cultura. A severidade da verrugose foi avaliada aos 84-92 dias do plantio, com escala específica de notas de um a quatro, em função dos sintomas exibidos nas hastes e pecíolos. No controle monitorado houve redução de uma a três pulverizações e os triazóis foram mais eficientes que o clorotalonil, resultando em rendimentos próximos aos do tratamento com quatro pulverizações fixas. O tebuconazole propiciou maior redução na intensidade da mancha preta, enquanto que o difenoconazole destacou-se pela eficiência no controle da verrugose.

Palavras-chave: Cercosporidium personatum, Sphaceloma arachidis. controle químico, Arachis hypogaea.

\section{ABSTRACT \\ Efficiency of fungicides in the control of peanut late leaf spot and scab by monitoring method}

Four field trials were carried out in Ribeirão Preto and Pindorama, SP, Brazil (1996 and 1997) to evaluate the efficiency of fungicides in the control of late leaf spot (Cercosporidium personatum) and scab (Sphaceloma arachidis) in peanut (Arachis hypogaea), cultivar Tatu. The treatments consisted of: control, not sprayed; conventional control, with four sprayings of chlorothalonyl at 14-day intervals, starting at 41 to 43 days after planting; monitored control with chlorothalonyl, tebuconazole, difenoconazole and propiconazole, respectively, in the recommended dosages for the peanut crop. Monitoring consisted of doing the first spraying when 5 to $15 \%$ of the leaflets were infected by the late leaf spot and, the following sprayings, at a minimum of 14-day intervals between applications, after three days, consecutive or not, in which rain precipitations greater than $2.5 \mathrm{~mm}$ were registered, in periods of seven days. Late leaf spot severity was assessed at weekly intervals during the growing season with diagrammatic scales of infected leaf area. Scab severity was evaluated at 84-92 days after planting with a specific scale of notes, ranging from one to four, according to the symptoms exhibited in stems and petioles. The results showed that in the monitored control, where the number of sprayings was reduced to one to three, the triazole fungicides were more efficient than chlorothalonyl, resulting in pod yields close to the treatment where four fixed sprayings were made. Tebuconazole promoted the highest reductions in late leaf spot intensity and, difenoconazole showed outstanding efficiency in the control of scab.

\section{INTRODUÇÃO}

\footnotetext{
* Auxílio financeiro do CNPq

** Bolsista do CNPq
}

Na cultura do amendoim (Arachis hypogaea L.), a intensidade das doenças da parte aérea pode variar com a 
Eficiência de fungicidas no controle da mancha preta e verrugose...

localidade e as épocas de plantio (Moraes \& Godoy, 1995; 1997). No Estado de São Paulo, maior produtor nacional, a mancha preta [Cercosporidium personatum (Berk. \& Curtis) Deignton] é a mais severa entre as doenças foliares do amendoim (Moraes et al., 1994). A verrugose do amendoim causada por Sphaceloma arachidis Bit. \& Jenk. é bastante importante quando ocorre nos estágios iniciais da cultura e apesar de ser considerada uma doença importante para as condições de cultivo do Estado de São Paulo, não se tem informações específicas sobre as perdas em produção que esta doença acarreta (Moraes \& Godoy, 1997).

Embora as medidas culturais de controle possam reduzir a severidade das doenças na cultura do amendoim, o controle químico é necessário. Trabalhos mais recentes indicam que clorotalonil e alguns triazóis (propiconazole, tebuconazole, difenoconazole e cyproconazole) são os mais eficientes no controle das manchas preta e castanha (Lopes et al., 1993; Dario et al., 1994; Leite et al., 1994), além de apresentarem controle sobre outras manchas foliares.

Os fungicidas comumente usados para o controle das manchas preta e castanha nem sempre controlam eficientemente a verrugose. O captafol, produto não disponível no mercado, era recomendado como o fungicida mais eficiente para o controle da verrugose (CATI, 1983; Godoy et al., 1983). Outros fungicidas têm sido recomendados, embora sejam de menor eficiência. Mais recentemente, a introdução de novos produtos para o controle das cercosporioses pode permitir a utilização de fungicidas que apresentem também eficiência no controle da verrugose. Entre esses produtos, o difenoconazole foi relatado como eficiente no controle da mancha preta, mancha castanha e verrugose (Lopes et al., 1993; Leite et al., 1994; Dario et al., 1994).

Apesar da necessidade do controle químico das doenças foliares, é possível reduzir a dependência da cultura do amendoim por fungicidas (Moraes \& Godoy, 1997). Nas condições de São Paulo, sob as variações climáticas de cinco anos agrícolas, estimou-se para a cultivar Tatu, que o número necessário de pulverizações para o controle de mancha preta variou de uma a três (Pedro Júnior et al., 1994; Moraes et al., 1994; Moraes et al., 1997). Após os primeiros 45 dias do ciclo da cultura do amendoim, o início da mancha preta pode ser monitorado visando prolongar, quanto possível, o início das pulverizações. Os resultados mostram que o limite de 5 a $15 \%$ de folíolos infetados, obtidos por amostragens, pode ser usado como regra para indicar a data da primeira pulverização com fungicida (Moraes \& Godoy, 1995; Moraes et al., 1997).

Além de melhorar a eficiência do controle das doenças, os métodos de previsão das pulverizações devem levar em consideração a rapidez e facilidade de sua utilização. Para a mancha preta, a utilização da precipitação pluvial como alternativa aos dados de umidade relativa (Davis et al., 1993; Bailey et al., 1994, Pezzopane et al., 1998), representa uma simplificação do monitoramento. Pezzopane et al. (1996) verificaram que, para a cultivar Tatu, uma quantidade de chuva diária acima de $2,5 \mathrm{~mm}$ foi uma variável climática adequada para um método mais simples de previsão de doença. Segundo Pezzopane et al. (1998), com a indicação de pulverização após a ocorrência de dois a quatro dias com precipitação pluvial acima de $2,5 \mathrm{~mm}$, os resultados foram semelhantes ao tratamento com pulverizações fixas, tanto em relação à intensidade da doença como na produtividade da cultura.

Este trabalho foi realizado visando comparar a eficiência de fungicidas sistêmicos aplicados segundo o método de monitoramento (incidência da mancha preta e precipitação pluvial), em relação ao controle convencional com fungicida de proteção, no controle da mancha preta e da verrugose do amendoim.

\section{MATERIAL E MÉTODOS}

Os ensaios foram conduzidos em Ribeirão Preto (no cultivo das "águas" de 1995/96 e 1996/97 e no da "seca" de 1996) e em Pindorama (cultivo das "águas" de 1995/96), em delineamento experimental de blocos ao acaso, com seis tratamentos e quatro repetições. Cada parcela correspondia a quatro linhas de $5 \mathrm{~m}$ de comprimento, com espaçamento de 0,60 m entre linhas e 0,10 m entre plantas, sendo utilizadas sementes da cultivar Tatu. Os tratamentos comparados foram: $\mathrm{A}=$ Testemunha - sem controle da doença; $\mathrm{B}=$ controle convencional (CATI, 1983), com quatro pulverizações de clorotalonil, a cada 14 dias, iniciando entre 41 e 43 dias do plantio; $\mathrm{C} \mathrm{a} \mathrm{F}=$ a primeira pulverização foi determinada por monitoramento, isto é, quando a incidência da mancha preta (através de amostragens de 20 folíolos coletados por parcela realizadas a cada três dias, a partir de 40 dias do plantio) atingiu 5 a $15 \%$ dos folíolos e as demais caso haja a ocorrência de três dias, seguidos ou não, com precipitações maiores que 2,5 mm, registradas durante períodos de sete dias, conforme metodologia citada por Pezzopane et al. (1996) e Moraes et al. (1997). A incidência da mancha preta foi acompanhada através de amostragens de 20 folíolos coletados por parcela (nos tratamentos $\mathrm{C}$ a F) realizadas a cada três dias, a partir de 42 dias do plantio.

Os fungicidas utilizados, nas doses recomendadas para a cultura, foram: clorotalonil $(1.500 \mathrm{~g} / \mathrm{l}$ do princípio ativo, na formulação 500-SC por hectare) de, nos tratamentos B e $\mathrm{C}$; tebuconazole (100 g/l do princípio ativo, na formulação 200-CE), no D; difenoconazole (87,5 g/l do princípio ativo, na formulação 200-CE), no E; e propiconazole (125 g/l do princípio ativo, na formulação 250-CE), no $\mathrm{F}$.

Em casos de aviso (por incidência da doença ou precipitação pluvial) para as pulverizações, estas deveriam ser realizadas no máximo 48 h após o aviso, respeitando-se, no caso de novas pulverizações, o período mínimo de 14 dias entre as aplicações dos fungicidas. Nenhuma pulverização foi realizada após 90 dias do plantio

A quantificação do progresso da mancha preta, após ser registrado seu início, foi feita pela coleta semanal de 20 folíolos, nas duas linhas centrais de cada parcela (parte mediana das plantas), sendo a severidade obtida pela comparação com escalas diagramáticas de porcentagem de 


\section{S. A. Moraes et al.}

área foliar com sintomas (Moraes, 1987). Em cada experimento foram realizadas de seis a 12 avaliações de incidência ou severidade, iniciando aos 42 dias do plantio e terminando próximo à colheita. $\mathrm{A}$ intensidade da doença durante o ciclo da cultura foi estimada para cada parcela através da área sob a curva do progresso da doença - ASCPD (Moraes et al., 1997) e calculou-se, para cada ensaio, o índice relativo da intensidade da doença nos tratamentos, em relação à testemunha (baseado na ASCPD total obtida no tratamento testemunha, considerada igual a 100). Entre a $12^{\mathrm{a}}$ e $13^{\mathrm{a}}$ semana do plantio, exceto para o ensaio dois (Pindorama 1996 das “águas"), avaliou-se o nível médio de desfolha, em porcentagem, através de comparação visual com o enfolhamento remanescente na haste principal de 10-15 plantas por parcela.

Em função da ocorrência da verrugose nos ensaios, esta doença foi avaliada entre a $12^{\mathrm{a}}$ e $13^{\mathrm{a}}$ semana do plantio, através da escala de notas com base nos sintomas exibidos nas hastes e pecíolos, variando de um (ausência de sintomas) a quatro (hastes completamente retorcidas recobertas por lesões, pecíolos e nervuras das folhas severamente afetadas, plantas amarelecidas ou mortas), em 10 plantas por parcela (Soave et al., 1973, Moraes et al., 1978 e Moraes et al., 1983).

Após a colheita foi realizada a pesagem das vagens secas, para determinação do rendimento em casca $(\mathrm{kg} / \mathrm{ha})$, sendo estimado o aumento relativo de produção de cada tratamento, em relação à testemunha.

Para as análises estatísticas, os resultados das avaliações da mancha preta (ASCPD) e verrugose (notas) foram transformados em $\sqrt{\mathrm{x}}$. Os dados obtidos de todas as variáveis foram submetidos a análise de variância para cada ensaio, individualmente, e a uma análise conjunta dos ensaios, no delineamento estatístico fatorial de seis tratamentos, quatro locais e quatro blocos casualizados, sendo as médias comparadas pelo teste Tukey a $1 \%$ de probabilidade.

\section{RESULTADOS E DISCUSSÃO}

A análise conjunta acusou significância do teste $\mathrm{F}$ a $1 \%$ de probabilidade, para a interação tratamentos $\mathrm{X}$ ensaios, em todas as variáveis (Tabela 2). Desta forma, os resultados são aqui discutidos em cada ensaio (Tabela 1).

Apesar da significância da interação, optou-se por incluir, nas discussões, a análise comparativa dos tratamentos, na média dos quatro ensaios (Tabela 2). Isso visa ilustrar a aplicação prática do método de monitoramento em estudo, e a sua eficiência média ao longo de diversos ambientes (anos e/ou locais).

A primeira pulverização dos diferentes tratamentos indicada pelo monitoramento inicial da mancha preta $(5$ a $15 \%$ de incidência) ocorreu entre 56 e 64 dias após o plantio (dap) nos ensaios das águas e aos 44 no ensaio da "seca", de Ribeirão Preto (Tabela 1).

O número de pulverizações nos tratamentos monitorados foi sempre menor (um a três) que no tratamento com pulverizações em datas fixas, sendo a primeira pulverização realizada 13 a 23 dias mais tarde que no tratamento com data preestabelecida (42 dap), exceto no ensaio da "seca", cuja indicação para pulverizar praticamente coincidiu com a primeira do tratamento com datas fixas . Com exceção do ensaio 1, onde apenas uma pulverização (por aviso do início da doença) foi realizada, nos tratamentos monitorados, o número de pulverizações realizadas exclusivamente por aviso de precipitação pluvial variou de um a dois (Tabela 1).

No ensaio 3 ("seca" em Ribeirão Preto), as condições climáticas, de temperatura, umidade e chuvas nos meses de fevereiro e março (início do ciclo da cultura), favoreceram a incidência da mancha preta mais cedo e o aumento rápido da intensidade da doença na fase intermediária do ciclo da cultura. Nos ensaios 1, 2 e 4 (das "águas"), particularmente o n ${ }^{\circ}$ 1, de Ribeirão Preto - 1996, apesar da ocorrência de precipitações pluviais, as condições de temperatura e número de horas com alta umidade relativa do ar no início do ciclo (35 a 50 dias do plantio) não se mostraram favoráveis à incidência da doença, causando o atraso no aparecimento dos primeiros sintomas da mancha preta e, conseqüentemente, na indicação da primeira pulverização com os fungicidas, com base na observação de 5 a $15 \%$ de folíolos com sintomas.

A intensidade da mancha preta, medida pela ASCPD durante o ciclo, apresentou diferenças significativas entre os ensaios, devido às condições climáticas registradas, sendo a maior intensidade verificada em Ribeirão Preto, no ensaio de 1996-seca, em função da incidência mais cedo da doença, seguida por Ribeirão Preto 1996 - “águas”.

A redução do número de pulverizações e a eficiência da associação do método de monitoramento do início da incidência da mancha preta com o de precipitação pluvial confirmam os resultados obtidos por Moraes et al. (1997). A recomendação de pulverização após a ocorrência de três dias com precipitação pluvial maior que $2,5 \mathrm{~mm}$, (Pezzopane et al., 1998), mostrou-se tão eficiente quanto ao tratamento com pulverizações fixas, tanto em relação à intensidade da doença como à produtividade.

Para cada ensaio (Figura 1), a evolução da intensidade da mancha preta nos tratamentos não pulverizados (NP) e com quatro pulverizações em datas fixas (FIX), em \% de ASCPDP (área sob a curva de progresso da doença proporcional à ASCPD total obtida para cada tratamento testemunha) e as ocorrências diárias de precipitações pluviais superiores a 2,5 mm ("dias chuvosos"). Em Ribeirão Preto 1996 - "águas" foram registradas 30 ocorrências de "dias chuvosos", durante o período de 42 a 105 dias do plantio (dap), sendo que a maioria delas (20) ocorreu até 75 dap. Considerando-se que a primeira pulverização indicada por incidência da mancha preta foi feita aos 64 dap e que no intervalo de 75 a 90 dap não foram registradas ocorrências de dias chuvosos, não foram indicados avisos de novas pulverizações com fungicidas (Figura 1).

No ensaio de Pindorama 1996 - “águas” ocorreram 25 registros de precipitações acima de $2,5 \mathrm{~mm}$, intercaladas com ausência de chuvas durante o período de 40 a 96 dap. No ensaio da "seca" de Ribeirão Preto foram registrados apenas 18 “dias chuvosos”, sendo 14 até 75 dap. Já no ensaio 
Eficiência de fungicidas no controle da mancha preta e verrugose...

TABELA 1 - Épocas de pulverizações e eficiência de fungicidas, em função das avaliações de mancha preta, verrugose, desfolha e rendimento em quatro ensaios (médias de 4 repetições) em amendoim (Arachis hypogaea)

\begin{tabular}{|c|c|c|c|c|c|c|c|c|}
\hline \multirow{2}{*}{ Trat. $^{1}$} & \multirow{2}{*}{$\begin{array}{l}\text { Pulverização } \\
\text { (Dap) }\end{array}$} & \multicolumn{2}{|c|}{ Mancha Preta $^{3}$} & \multicolumn{2}{|c|}{ Verrugose $^{4}$} & \multirow{2}{*}{$\begin{array}{l}\text { Desfolha } \\
(\%)^{5}\end{array}$} & \multicolumn{2}{|c|}{ Rendimento $^{6}$} \\
\hline & & ASCPD & Índice & Nota & Índice & & $\mathrm{Kg} / \mathrm{ha}$ & Índice \\
\hline \multicolumn{9}{|c|}{ Ensaio 1 - Ribeirão Preto 1996 "águas" } \\
\hline A & Nenhuma & $9,67 \mathrm{a}$ & 100 & 1,92 a & 100 & $67,5 \mathrm{a}$ & $2227 \mathrm{bc}$ & 100 \\
\hline $\mathrm{B}$ & $41-55-70-85$ & $7,12 \mathrm{~b}$ & 74 & $1,89 \mathrm{a}$ & 98 & $20,0 \mathrm{~b}$ & $2757 \mathrm{ab}$ & 124 \\
\hline $\mathrm{C}$ & 64 & $7,11 \mathrm{~b}$ & 74 & $1,80 \mathrm{ab}$ & 94 & $32,5 \mathrm{~b}$ & $2190 \mathrm{c}$ & 98 \\
\hline $\mathrm{D}$ & 64 & $5,06 \mathrm{~b}$ & 52 & $1,62 \mathrm{bc}$ & 84 & $20,0 \mathrm{~b}$ & $2508 \mathrm{abc}$ & 113 \\
\hline $\mathrm{E}$ & 64 & $5,89 \mathrm{~b}$ & 61 & $1,44 \mathrm{c}$ & 75 & $17,5 \mathrm{~b}$ & $2817 \mathrm{a}$ & 126 \\
\hline \multirow[t]{3}{*}{$\mathrm{F}$} & 64 & $6,95 \mathrm{~b}$ & 72 & $1,63 \mathrm{bc}$ & 85 & $30,0 \mathrm{~b}$ & $2714 \mathrm{abc}$ & 122 \\
\hline & $\mathrm{CV} \%$ & 14,32 & & 5,48 & & 25,85 & 9,44 & \\
\hline & S. Tukey $1 \%\left({ }^{7}\right)$ & 2,29 & & 0,22 & & 18,58 & 550,82 & \\
\hline \multicolumn{9}{|c|}{ Ensaio 2 - Pindorama 1996 "águas" } \\
\hline A & Nenhuma & $5,73 \mathrm{a}$ & 100 & $1,87 \quad \mathrm{a}$ & 100 & n. a. & $3228 \mathrm{~b}$ & 100 \\
\hline $\mathrm{B}$ & $43-58-71-85$ & $1,03 \mathrm{~b}$ & 18 & $1,83 \mathrm{ab}$ & 98 & n. a . & $4660 \mathrm{a}$ & 144 \\
\hline $\mathrm{C}$ & $56-86$ & $1,78 \mathrm{~b}$ & 31 & $1,85 \mathrm{ab}$ & 99 & n. a . & $4218 \mathrm{a}$ & 131 \\
\hline $\mathrm{D}$ & $56-86$ & $1,28 \mathrm{~b}$ & 22 & $1,71 \mathrm{ab}$ & 91 & n. a . & $4860 \mathrm{a}$ & 150 \\
\hline $\mathrm{E}$ & $56-86$ & $0,95 \mathrm{~b}$ & 17 & $1,17 \mathrm{c}$ & 63 & n. a . & $5041 \mathrm{a}$ & 156 \\
\hline \multirow[t]{3}{*}{$\mathrm{F}$} & $56-86$ & $1,03 \mathrm{~b}$ & 18 & $1,51 \mathrm{bc}$ & 81 & n. a. & $4307 \mathrm{a}$ & 133 \\
\hline & $\mathrm{CV} \%$ & 26,47 & & 9,23 & & & 9,43 & \\
\hline & .S. Tukey $1 \%\left({ }^{7}\right)$ & 1,20 & & 0,35 & & & 951,16 & \\
\hline \multicolumn{9}{|c|}{ Ensaio 3 - Ribeirão Preto 1996 "seca". } \\
\hline A & Nenhuma & $12,13 \mathrm{a}$ & 100 & $2,00 \mathrm{a}$ & 100 & 65,0 a & $631 \mathrm{~b}$ & 100 \\
\hline $\mathrm{B}$ & $43-57-71-85$ & $8,26 \mathrm{bc}$ & 68 & $1,73 \mathrm{~b}$ & 87 & $35,0 \mathrm{bc}$ & $1200 \mathrm{a}$ & 190 \\
\hline $\mathrm{C}$ & 44-65-85 & $10,30 \mathrm{ab}$ & 85 & $1,80 \mathrm{ab}$ & 90 & $52,5 \mathrm{ab}$ & $950 \mathrm{ab}$ & 150 \\
\hline $\mathrm{D}$ & $44-65-85$ & $5,51 \mathrm{c}$ & 45 & $1,62 \mathrm{~b}$ & 81 & $35,0 \mathrm{bc}$ & $1130 \mathrm{ab}$ & 179 \\
\hline $\mathrm{E}$ & $44-65-85$ & $8,41 \mathrm{~b}$ & 69 & $1,36 \mathrm{c}$ & 68 & $22,5 \mathrm{c}$ & $1069 \mathrm{ab}$ & 169 \\
\hline \multirow[t]{3}{*}{$\mathrm{F}$} & $44-65-85$ & $8,52 \mathrm{~b}$ & 70 & $1,65 \mathrm{~b}$ & 83 & $47,5 \mathrm{ab}$ & $1161 \mathrm{a}$ & 184 \\
\hline & $\mathrm{CV} \%$ & 14,02 & & 6,42 & & 18,17 & 21,70 & \\
\hline & .S. Tukey $1 \%\left({ }^{7}\right)$ & 2,85 & & 0,25 & & 17,94 & 510,78 & \\
\hline \multicolumn{9}{|c|}{ Ensaio 4 - Ribeirão Preto 1997 "águas" } \\
\hline A & Nenhuma & $3,32 \mathrm{ab}$ & 100 & $1,93 \mathrm{a}$ & 100 & 82,5 a & $871 \mathrm{c}$ & 100 \\
\hline $\mathrm{B}$ & $42-56-70-84$ & $2,96 \mathrm{abc}$ & 89 & $1,66 \mathrm{bc}$ & 86 & $21,3 \mathrm{~d}$ & $2414 \mathrm{a}$ & 277 \\
\hline $\mathrm{C}$ & $57-72-89$ & $3,80 \mathrm{a}$ & 114 & $1,80 \mathrm{ab}$ & 93 & $65,0 \mathrm{~b}$ & $1294 \mathrm{bc}$ & 149 \\
\hline $\mathrm{D}$ & $57-72-89$ & $2,09 \mathrm{c}$ & 63 & $1,66 \mathrm{bc}$ & 86 & $45,0 \mathrm{c}$ & $1565 \mathrm{abc}$ & 180 \\
\hline $\mathrm{E}$ & $57-72-89$ & $3,01 \mathrm{ab}$ & 91 & $1,50 \mathrm{c}$ & 78 & $26,3 \mathrm{~d}$ & $1943 \mathrm{ab}$ & 223 \\
\hline $\mathrm{F}$ & $57-72-89$ & $2,77 \mathrm{bc}$ & 83 & $1,66 \mathrm{bc}$ & 86 & $55,0 \mathrm{bc}$ & $1585 \mathrm{abc}$ & 182 \\
\hline \multirow{2}{*}{$\Gamma$} & $\mathrm{CV} \%$ & 13,11 & & 6,65 & & 12,36 & 23,28 & \\
\hline & Tukey $1 \%\left({ }^{7}\right)$ & 0,90 & & 0,23 & & 13,98 & 863,25 & \\
\hline
\end{tabular}

${ }^{1}$ Tratamentos com clorotalonil em quatro pulveizações em datas fixas (B), pulverizações monitoradas com clorotalonil (C), tebuconazole (D), difenoconazole (E) e propiconazole $(\mathrm{F})$,

2 Época de realização das pulverizações em dias após o plantio (dap).

${ }^{3}$ Valores de área sob a curva de progresso da doença (ASCPD transformados em $\sqrt{\mathrm{x}}$ e índice relativo à testemunha.

${ }^{4}$ Valores de escala de notas variando entre 1 a 4 , transformados em $\sqrt{ } \mathrm{x}$ e índice relativo à testemunha.

${ }^{5}$ Avaliada na $13^{\text {a }}$ semana após o plantio.

${ }^{6} \mathrm{Kg}$ de amendoim em casca por hectare e índice relativo à testemunha.

${ }^{7}$ Médias de ASCPD, verrugose, desfolha ou rendimento dentro de cada ensaio seguidas por letras iguais não diferem entre si, pelo teste Tukey a $1 \%$.

quatro ocorreram 37 dias chuvosos bem distribuídos no período de 43 a 105 dap.

A análise conjunta dos ensaios não detectou diferença significativa entre os ensaios para as avaliações de severidade da verrugose (Tabela 2). Porém, mesmo com o uso de métodos de monitoramento baseados na mancha preta, foi possível verificar diferenças no controle dessa doença, com uma a três pulverizações com os fungicidas sistêmicos, com o difenoconazole se destacando em todos os ensaios, tendo o tebuconazole e o propiconazole comportamento intermediário e o clorotalonil apresentando baixa eficiência no seu controle. Os resultados obtidos confirmam as avaliações feitas por Lopes et al. (1993); Leite et al. (1994); Dario et al. (1994), sobre a ação desse produto (difenoconazole) no controle da verrugose, 
TABELA 2 - Número de pulverizações, intensidade da mancha preta, severidade da verrugose e rendimento de amendoim (Arachis hypogaea), em cada tratamento, e resumo da análise de variância conjunta das médias dos quatro ensaios

\begin{tabular}{|c|c|c|c|c|c|c|c|c|}
\hline \multirow{2}{*}{$\operatorname{Trat}^{1}$} & \multirow{2}{*}{$\begin{array}{c}\mathbf{N}^{0} \text { de } \\
\text { Pulverizações }\end{array}$} & \multicolumn{2}{|c|}{ Mancha Preta } & \multicolumn{2}{|c|}{ Verrugose } & \multirow{2}{*}{$\begin{array}{c}\text { Desfolha } \\
(\%)\end{array}$} & \multicolumn{2}{|c|}{ Rendimento } \\
\hline & & ASCPD & $\mathrm{C} \%{ }^{2}$ & Notas & $\mathrm{C} \%{ }^{2}$ & & $\mathrm{Kg} / \mathrm{Ha}$ & $G \%{ }^{3}$ \\
\hline A & Nenhuma & $7,71 \quad a$ & -- & $1,93 \mathrm{a}$ & - & $72 \mathrm{a}$ & $1739 \mathrm{c}$ & -- \\
\hline B & 4 & $4,84 \mathrm{c}$ & 37 & $1,78 \mathrm{~b}$ & 8 & $25 \mathrm{~cd}$ & 2758 a & 58 \\
\hline $\mathrm{C}$ & 1 a 3 & $5,74 \quad b$ & 25 & $1,82 \mathrm{ab}$ & 6 & $50 \mathrm{~b}$ & $2163 \mathrm{c}$ & 24 \\
\hline $\mathrm{D}$ & 1 a 3 & $3,49 \mathrm{~d}$ & 54 & $1,65 \mathrm{c}$ & 14 & $33 \mathrm{c}$ & 2516 a & 45 \\
\hline E & 1 a 3 & $4,57 \mathrm{c}$ & 41 & $1,37 \mathrm{~d}$ & 29 & $22 \mathrm{~d}$ & 2718 a & 56 \\
\hline $\mathrm{F}$ & 1 a 3 & $4,82 \mathrm{c}$ & 37 & $1,61 \mathrm{c}$ & 17 & $44 \mathrm{~b}$ & $2442 a b$ & 40 \\
\hline DMS & Tukey $1 \%\left({ }^{4}\right)$ & 0,87 & & 0,12 & & 8,47 & 343,72 & \\
\hline \multirow{3}{*}{$\begin{array}{c}\text { Teste F } \\
\left({ }^{5}\right)\end{array}$} & Tratamentos (T) & 46,64 & ** & 48,75 & ** & $82,46 * *$ & 21,41 & ** \\
\hline & Ensaios (E) & 363,29 & $* *$ & 1,22 & Ns & $40,36 * *$ & 470,72 & ** \\
\hline & $\mathrm{T} \times \mathrm{E}$ & 5,69 & $* *$ & 2,38 & $* *$ & $4,45 * *$ & 2,82 & $* *$ \\
\hline \multirow[b]{2}{*}{ Média } & CV \% & 16,11 & & 6,65 & & 17,06 & 13,88 & \\
\hline & geral dos ensaios & 5,19 & & 1,69 & & 41,11 & 2389,22 & \\
\hline
\end{tabular}

${ }^{1}$ Tratamentos com clorotalonil em 4 pulveizações em datas fixas (B), pulverizações monitoradas com clorotalonil $(\mathrm{C})$, tebuconazole $(\mathrm{D})$, difenoconazole $(\mathrm{E})$ e propiconazole $(\mathrm{F})$,

${ }^{2} \mathrm{C} \%=\%$ de controle da intensidade da doença, em relação à testemunha não pulverizada com fungicida.

${ }^{3} \mathrm{G} \%=\%$ de ganho em rendimento em relação à testemunha.

${ }^{4}$ Médias de ASCPD e verrugose, transformadas em $V_{\mathrm{x}}$, ou de rendimento dos 4 ensaios, seguidas por letras iguais, não diferem entre si pelo teste Tukey a $1 \%$.

${ }^{5}$ Resumo da análise de variância conjunta dos 4 ensaios, para cada variável.

que pode ser indicado como uma boa alternativa para substituir o captafol, que era considerado como o fungicida mais eficiente no controle dessa doença.

A verrugose é uma doença que leva à diminuição do crescimento das plantas, por causar hipertrofia e hiperplasia das hastes e pecíolos das folhas, enquanto que as manchas foliares são as principais responsáveis pela desfolha nas plantas (Moraes \& Godoy, 1997). Nesse sentido, a desfolha verificada nos ensaios pode ser atribuída principalmente à mancha preta, trazendo como conseqüência a diminuição na duração da área foliar sadia e no rendimento da cultura (Aquino et al., 1992), especialmente quando não controlada com eficiência .

O nível de desfolha na ausência de controle químico foi maior especialmente nos ensaios 1 e 4 (Tabela 1). No ano agrícola de 1996/97, em Ribeirão Preto (ensaio 4), o aparecimento mais cedo e com alta intensidade da mancha castanha, fato não comum nos últimos anos naquele local (por isso não foi avaliada individualmente), levou à desfolha precoce nos tratamentos não pulverizados com fungicidas (82,5\% de desfolha aos 90 dias do plantio), afetando de maneira drástica a produtividade, com queda de produção superior a $100 \%$ em relação aos tratamentos que se destacaram (quatro pulverizações em datas fixas com clorotalonil e três pulverizações monitoradas com difenoconazole), indicando também a ação dos fungicidas sobre essa doença. Além disso, a desfolha precoce das plantas infetadas resultou em baixa intensidade da mancha preta, não havendo diferenças significativas entre os tratamentos nesse ensaio (Tabela 1).

As produtividades médias variaram significativamente entre os ensaios (Tabela 2). A maior produtividade foi observada no ensaio 2, de Pindorama - 1996 das "águas" (média do ensaio de $4385 \mathrm{~kg} / \mathrm{ha}$ ) e a menor no ensaio 3, da "seca" de Ribeirão Preto - 1996 (média do ensaio de 1024 kg/ha).

Os rendimentos obtidos nos tratamentos, em $\mathrm{kg}$ de amendoim em casca por hectare, apresentaram diferenças significativas, em todos os ensaios. Os tratamentos com pulverizações monitoradas, exceto para o clorotalonil nos ensaios 1 e 4, apresentaram rendimentos que não diferiram significativamente do tratamento com quatro pulverizações em datas fixas com clorotalonil. Na ausência de controle químico das doenças os rendimentos foram menores (Tabela 1).

Os resultados da análise conjunta dos ensaios mostram que no controle monitorado (com redução de uma a três pulverizações), os triazóis foram mais eficientes que o clorotalonil, resultando em rendimentos que não diferiram significativamente do tratamento com quatro pulverizações fixas, apresentando ganhos de 40 a $56 \%$ em relação ao tratamento não pulverizado com fungicidas. O tebuconazole (tratamento D) propiciou maior redução na intensidade da mancha preta (54\%) e baixa desfolha (33\%), enquanto que o difenoconazole destacou-se pela eficiência no controle da verrugose e menor desfolha das plantas (Tabela 2).

Labrinos \& Nutter (1993), estudando o efeito do clorotalonil e do tebuconazole sobre a mancha preta, 


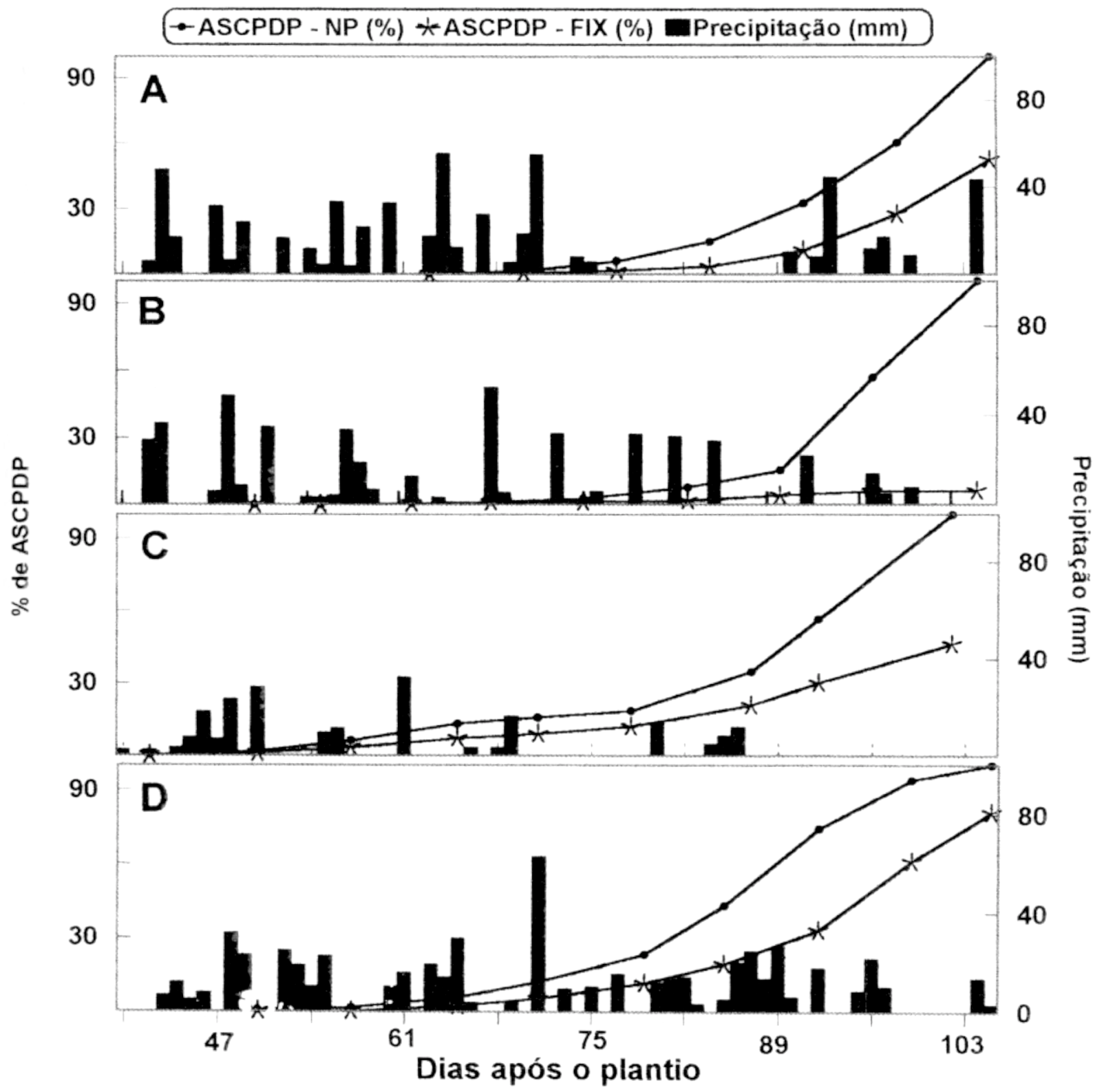

FIG. 1 - Evolução da intensidade da mancha preta do amendoim (Arachis hypogaea) nos tratamentos não pulverizado (\% de ASCPDP-NP) e com quatro pulverizações em datas fixas (\% de ASCPDP-FIX), baseadas na ASCPD total obtida no tratamento testemunha, e ocorrências diárias de precipitações pluviais superiores a 2,5 mm, nos ensaios: A (Ensaio 1 - Ribeirão Preto 1996 “águas"), B (Ensaio 2 - Pindorama 1996 "águas"), C (Ensaio 3 - Ribeirão Preto 1996 “seca”) e D (Ensaio 4 - Ribeirão Preto 1997 “águas”).

verificaram que ambos reduzem a germinação dos esporos e a taxa de esporulação nas lesões, mas apenas o tebuconazole é capaz de reduzir a freqüência de infecção, diminuir o tamanho das lesões e aumentar o período de incubação, após os tubos germinativos terem penetrado nas folhas do amendoim. No presente trabalho, a diferença obtida entre esses fungicidas foi expressa pela ASCPD, que leva em consideração o número e tamanho das lesões (área com sintomas) e a evolução da doença durante o ciclo.

Em função das suas características (Labrinos \& Nutter, 1993) e dos resultados obtidos nesse trabalho, o clorotalonil poderia ser utilizado em aplicações alternadas com fungicidas sistêmicos, como o tebuconazole ou o difenoconazole, quando a severidade das doenças foliar permitisse ou exigisse o uso de fungicidas de proteção ou sistêmicos.

Os resultados obtidos sugerem ser possível estabelecer medidas de manejo integrado das doenças do amendoim, por meio do acompanhamento da incidência e severidade de cada doença, de modo a indicar quando pulverizar, o tipo de fungicida mais adequado para cada caso ou a alternância entre fungicidas de proteção e de ação sistêmica, visando a eficiência no controle e a redução de perdas na produção. 


\section{REFERÊNCIAS BIBLIOGRÁFICAS}

AQUINO, V.M., SHOKES, F.M., BERGER, R.D., GORBET, D.W. \& KUCHAREK, T.A. Relantionships among late leafspot, healthy leaf area duration, canopy refletance, and yield of peanut. Phytopathology 82:546-552.1992.

BAILEY, J.E., JOHNSON, G.L. \& TOTH JR, S.J. Evolution of a weather-based peanut leaf spot advisory in North Carolina. Plant Disease 78:530-535.1994.

COORDENADORIA DE ASSISTÊNCIA TÉCNICA INTEGRAL (CATI). Controle de pragas e doenças da cultura do amendoim. Campinas: CATI, Boletim Técnico 174, 1983.

DARIO, G.J.A., LEITE, O.M.C. \& DARIO, P.W. Avaliação da eficiência do difenoconazole no controle de fungos que atacam a parte aérea do amendoim. Fitopatologia Brasileira 19:283. 1994 (Resumo).

DAVIS, D.P., JACOBI, J.C. \& BACKMAN, P.A. Twentyfour-hour rainfall, a simple environmental variable for predicting peanut leaf spot epidemics. Plant Disease 77:722-725.1993.

GODOY, J.R., GIMENES-FERNANDES, N. \& KRONKA, S.N. Controle de doenças foliares do amendoim: eficiência de fungicidas e influência na produtividade. Summa Phytopathologica 9:196-206.1983.

LABRINOS, J.L. \& NUTTER JR., F.W. Effects of a protectant versus a systemic fungicide on disease components of late leaf spot. Plant Disease 77:837-845.1993.

LEITE, O.M.C., DE VICENZO, M.C.V. \& BALTIERI, E.M. Avaliação da eficiência do difenoconazole no controle de fungos que atacam a parte aérea do amendoim. Fitopatologia Brasileira 19:274-275. 1994. (Resumo)

LOPES, M.E.B.M., LASCA, D.H.C., GUILHEM, D.J., MONTES, S.M.N.M., CEZARIO, A.C. \& CERAVOLO, L.C. Controle das doenças foliares do amendoim (Arachis hypogaea L.). Fitopatologia Brasileira 18:301.1993. (Resumo).

MORAES, S.A. Escalas diagramáticas para avaliar a intensidade das cercosporioses do amendoim. O Agronômico 39:9-18.1987.

MORAES, S.A. \& GODOY, I.J. Controle integrado de doenças do amendoim. Mesa redonda: Controle integrado de doenças em culturas de importância econômica. XVIII Congresso Paulista de Fitopatologia, Piracicaba, SP. Summa Phytopathologica 21:63-64. 1995.
MORAES, S.A. \& GODOY, I.J. Amendoim - Controle de doenças. In: Vale, F.X.R. \& Zambolim, L. (Eds.) Controle de Doenças de Plantas: grandes Culturas, Viçosa, MG. UFV. Suprema Gráfica e Editora Ltda. 1997. pp. 1-49.

MORAES, S.A., GODOY, I.J. \& GERIN, M.A.N. Avaliação de resistência de Arachis hypogaea a Puccinia arachidis, Sphaceloma arachidis e Phoma arachidicola. Fitopatologia Brasileira 8:499-506. 1983.

MORAES, S.A., SOAVE, J. \& SAVY FILHO, A. Reações de 10 variedades de amendoim (Arachis hypogaea L.) à verrugose (Sphaceloma arachidis Bit. \& Jenk.). Ecossistema 3:43-46. 1978.

MORAES, S.A., GODOY, I.J., MARTINS, A.L.M., PEREIRA, J.C.V.N.A. \& PEDRO JÚNIOR., M.J. Epidemiologia da mancha preta (Cercosporidium personatum) em amendoim: resistência, controle químico e progresso da doença. Fitopatologia Brasileira 19:532-540. 1994.

MORAES, S.A., GODOY, I.J., PEDRO JÚNIOR., M.J, MARTINS, A.L.M., PEREIRA, J.C.V.N.A. \& PEZZOPANE, J.R.M. Monitoramento da mancha preta associado a parâmetros climáticos para predizer a necessidade de controle químico em amendoim. Fitopatologia Brasileira 22:419-426. 1997.

PEDRO JÚNIOR, M. J., MORAES, S.A.; \& GODOY, I.J. Agrometeorological forecasting method for Cercospora leafspot in peanuts. Fitopatologia Brasileira 19:69-73. 1994.

PEZZOPANE, J.R.M., PEDRO JÚNIOR, M.J., SENTELHAS, P.C., MORAES, S.A. \& GODOY, I.J. Uso da precipitação para previsão de mancha de cercospora em amendoim. Fitopatologia Brasileira 21:426-430. 1996.

PEZZOPANE, J.R.M., PEDRO JÚNIOR., M.J, MORAES, S.A., GODOY, I.J., PEREIRA, J.C.V.N.A. \& SILVEIRA, L.C.P. Chuva e previsão de épocas de pulverização para controle das manchas foliares do amendoim. Bragantia 57:285-295. 1998

SOAVE, J., PARADELA FILHO, O., RIBEIRO., I.J.A., SUGIMORI, M.H., SAVY FILHO, A., CANECCHIO FILHO, V. \& NELLI, E.J. Avaliação da resistência de variedade de amendoim (Arachis hypogaea L.) a verrugose (Sphaceloma arachidis Bit. \& Jenk.) em condições de campo. Revista de Agricultura 48:129-132. 1973. 